\title{
No essential role for tripeptidyl peptidase II for the processing of LCMV-derived T cell epitopes
}

\author{
Michael Basler ${ }^{1}$ and Marcus Groettrup ${ }^{1,2}$ \\ ${ }^{1}$ Division of Immunology, Department of Biology, University of Constance, Konstanz, \\ Germany \\ ${ }^{2}$ Biotechnology Institute Thurgau, Tägerwilen, Switzerland
}

The proteasome is critically involved in the production of MHC class I-restricted T cell epitopes. Approximately $20 \%$ of all peptides generated by the proteasome are too large for direct presentation by MHC class I molecules. Reits et al. (Immunity 2004. 20: 495-506) suggested that a major portion of proteasomal products are larger than 15 amino acids and require further degradation by the tripeptidyl peptidase II (TPPII) before becoming ligands of MHC class I molecules. Using the well-characterized lymphocytic choriomeningitis virus (LCMV) model, the role of TPPII in the processing of several LCMV-derived T cell epitopes was investigated. In contrast to Reits' proposal, TPPII inhibition and TPPII overexpression experiments revealed that five out of six LCMV-derived $\mathrm{CD} 8^{+} \mathrm{T}$ cell epitopes were not affected by inhibition of TPPII, while one epitope (GP276) was slightly reduced upon TPPII overexpression. Additionally, we demonstrated that the processing of two epitopes derived from ovalbumin and murine cytomegalovirus were not altered by TPPII inhibition. We propose that TPPII is not generally required for the production of MHC class I peptides, but the presentation of some peptides can be negatively affected by TPPII.

Key words:

Antigen processing Cytotoxic T cells

- Proteasome

\section{Introduction}

The major histocompatibility complex (MHC) class Irestricted pathway of antigen processing allows the presentation of intracellular antigens to cytotoxic T lymphocytes. The generation of small peptides of 8-10 amino acids (aa) fitting into the groove of MHC

Correspondence: Dr. Michael Basler, Department of Biology, Division of Immunology, University of Constance, P1101 Universitätsstrasse 10, D-78457 Konstanz, Germany Fax: +49-7531-883102

e-mail: Michael.Basler@uni-konstanz.de

Abbreviations: AAF-AMC: Ala-Ala-Phe-7-amido-

4-methylcoumarin - AAF-CMK: Ala-Ala-Phe-chloromethyl

ketone - BFA: brefeldin A - BH: bleomycin hydrolase

E:S ratio: effector-to-stimulator ratio - GP: glycoprotein .

ICS: intracellular cytokine staining · LCMV: lymphocytic

choriomeningitis virus - MCMV: murine cytomegalovirus

NP: nucleoprotein · PSA: puromycin-sensitive

aminopeptidase · tet: tetracycline - TPPII: tripeptidyl

peptidase II $\cdot \mathbf{r V V}$ : recombinant vaccinia virus class I molecules results from a multi-step process. Proteins are degraded by the ubiquitin-proteasome system, leading to the release of peptides from the proteasome that range in size from 2 to 25 aa [1]. Peptides can be further trimmed or destroyed by cytosolic proteases like leucine aminopeptidase (LAP) [2], bleomycin hydrolase (BH) [3], puromycin-sensitive aminopeptidase (PSA) [3], thimet oligopeptidase (TOP) $[4,5]$, and tripeptidyl aminopeptidase II (TPPII) [6-8]. Cytosolic peptides designated for MHC class I presentation are then transported into the lumen of the endoplasmic reticulum (ER) via the transporter associated with antigen processing. In the $\mathrm{ER}$, further $\mathrm{N}$-terminal trimming of peptides can occur by the ERassociated aminopeptidases ERAAP or ERAP1/2 [9-11]. Once the MHC class I molecule binds an appropriate peptide, it is released from the MHC class-I loading complex and rapidly transported via the Golgi apparatus to the cell surface, where it presents the loaded peptide to cytotoxic $\mathrm{T}$ cells. 
The proteasome plays a critical role in the generation of the majority of MHC class I-presented peptides. It has been estimated that approximately $60 \%$ of proteasomegenerated fragments are too small, $15 \%$ are of the appropriate size and $20 \%$ are too large for direct presentation by MHC class I [12]. This implies that most of the longer peptides need to be further trimmed before properly fitting MHC class I molecules. Reits et al. [8] reported that TPPII is required for trimming the proteasome-derived precursor of most MHC class I ligands. In mammals, TPPII consists of several 138-kDa subunits building a large homooligomeric amino peptidase belonging to the subtilisin family. High-resolution cryo-electron microscopy of the TPPII homologue from fruit fly revealed that the building blocks of this large complex are head-to-head-oriented dimers. Stacking of these dimers leads to the formation of two twisted strands [13]. TPPII removes tripeptides from the $\mathrm{N}$ terminus of longer peptides, but also a low endopeptidase activity has been demonstrated [6], implying a potential function for TPPII in the generation of ligands for MHC class I molecules. In fact, it has been demonstrated that TPPII is crucial for the generation of the HIV epitope Nef(73-82) in a proteasome-independent manner [7] and for the production of the RU1(31-42) epitope from $\mathrm{N}$-extended precursors [14]. In order to clarify the role for TPPII in the generation of virus-derived $\mathrm{T}$ cell epitopes, we used the lymphocytic choriomeningitis virus (LCMV) and investigated the contribution of TPPII in the production of several LCMVderived ligands for MHC class I molecules.

\section{Results}

\section{LCMV-derived NP118-126 presentation is independent of TPPII}

In order to investigate the role of the cytosolic subtilisinlike TPPII in the processing of the LCMV-derived T cell epitope nucleoprotein (NP)118-126, we infected J774 cells $\left(\mathrm{H}-2^{\mathrm{d}}\right)$ with LCMV-WE and treated the cells with the TPPII inhibitor Ala-Ala-Phe-chloromethyl ketone (AAF-CMK; 20 and $40 \mu \mathrm{M}$ ). The amount of peptides was detected with NP118-126-specific CTL by doublestaining for CD8 and intracellular IFN- $\gamma$ [intracellular cytokine staining (ICS) for IFN- $\gamma$ ]. No difference in NP118 presentation between AAF-CMK-treated and untreated cells was observed (Fig. 1A). To confirm these results in a virus-independent model, we used a tetracycline (tet)-regulated system that relies on the tetrepressible expression of LCMV-NP in the fibroblast transfectant B8tNP64 [15]. In these cells, NP expression can be switched on by removing tet from the growth medium. Also in this virus-independent system, no effect of TPPII on the presentation of NP118 could be observed (Fig. 1B). Although AAF-CMK is a potent inhibitor of TPPII [16], it has been reported that this inhibitor can affect other proteases like PSA and BH [3, 14]. To corroborate the obtained findings, the more specific TPPII inhibitor butabindide was applied. Because serum factors can drastically reduce butabindide activity, experiments were performed with extensively washed cells in the absence of FCS [8]. Neither in LCMV-infected cells nor in cells infected with a vaccinia virus expressing the LCMV-NP (VVYN4) was a change of NP118 presentation in butabindide-treated cells compared to untreated cells observed (Fig. 1C, D).

\section{TPPII inhibition does not affect the presentation of LCMV-derived $\mathrm{T}$ cell epitopes}

To address whether other T cell epitopes were affected by TPPII, five different $\mathrm{H}-2 \mathrm{D}^{\mathrm{b}} / \mathrm{K}^{\mathrm{b}}$-restricted LCMV-derived epitopes were investigated. AAF-CMK- or butabindidetreated (Fig. 2A) BMC cells were infected with LCMVWE and the amount of peptides presented on MHC class I was detected with peptide-specific CTL lines against glycoprotein (GP)33-41/D ${ }^{\mathrm{b}}$ or $\mathrm{K}^{\mathrm{b}}$, GP276-286/

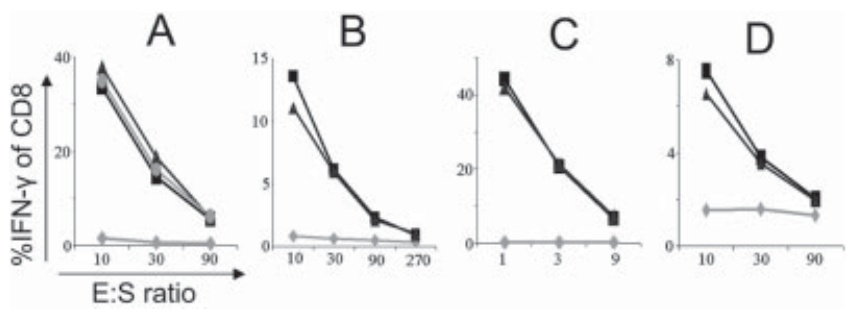

Figure 1. NP118-126 antigen presentation is not affected by TPPII inhibition. Detection of LCMV NP118-126 epitopes by splenocytes derived from LCMV-infected BALB/c mice. Activation of $\mathrm{T}$ cells by stimulator cells was analyzed by staining for CD8 and intracellular IFN- $\gamma$. Shown are the percentages of IFN- $\gamma$ positive cells of $\mathrm{CD}^{+}$cells as determined by flow cytometry. The percentage of intracellular IFN- $\gamma$ (y axis) produced by CTL lines is plotted versus the E:S ratio. (A) J774 cells were infected with LCMV-WE and treated with $20 \mu \mathrm{M}$ (triangles) or $40 \mu \mathrm{M}$ (squares) AAF-CMK overnight or left untreated (circles). Uninfected cells are marked with diamonds. One experiment out of three is shown. (B) After inducing LCMV-NP expression in B8tNP64 cells by removing tet, cells were treated for $24 \mathrm{~h}$ with $20 \mu \mathrm{M}$ (triangles) AAF-CMK or left untreated (squares) before NP118 presentation was assessed. Not induced cells are marked with diamonds. (C) LCMV-infected J774 cells were treated with $100 \mu \mathrm{M}$ (triangles) butabindide overnight or left untreated (squares) before addition of NP118-specific CTL. Uninfected cells are marked with diamonds. One experiment out of two is shown. (D) $J 774$ cells were infected with VVYN4 (encoding LCMV-NP) and treated with $100 \mu \mathrm{M}$ (triangles) butabindide in the absence of FCS for $3 \mathrm{~h}$ or left untreated (squares) prior to determination of NP118 presentation. Uninfected cells are marked with diamonds. One experiment out of two is shown. 
$\mathrm{D}^{\mathrm{b}}, \mathrm{NP} 396-404 / \mathrm{D}^{\mathrm{b}}, \mathrm{NP} 205-212 / \mathrm{K}^{\mathrm{b}}$, and GP92-101/D $\mathrm{D}^{\mathrm{b}}$. AAF-CMK had no effect on the presentation of GP33, NP396, and NP205 and resulted in a slightly increased presentation of GP276 and GP92. Hence, TPPII and other proteases inhibited by AAF-CMK have no impact in generating these two epitopes, but rather have a minor destructive role. In order to further investigate the role of TPPII in the generation of LCMV-derived epitopes, the more specific TPPII inhibitor butabindide was applied. Butabindide inhibition slightly augmented the amount of GP276 presented on MHC class I but had no significant effect on the other epitopes. In order to confirm the inhibition of TPPII by AAF-CMK or
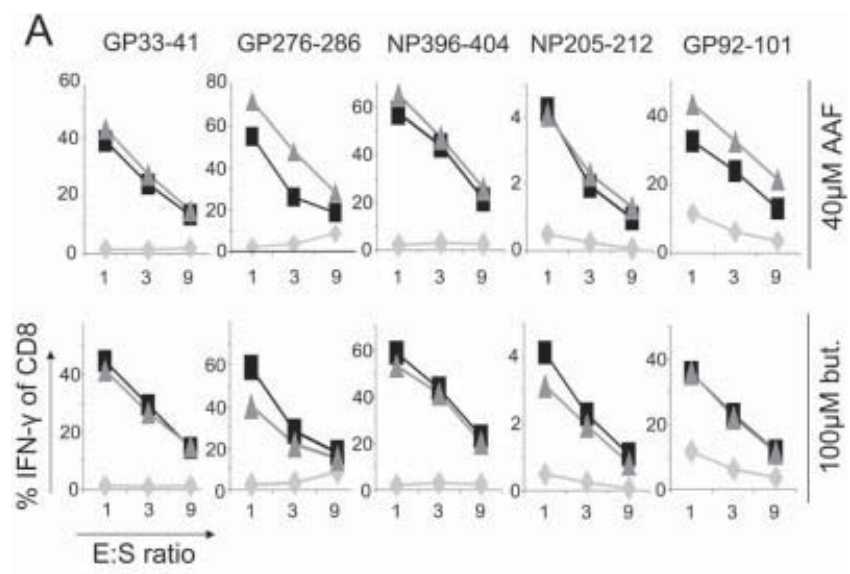

B

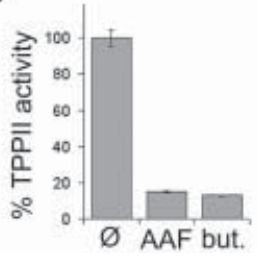

Figure 2. Marginal effect of TPPII inhibition on the presentation of LCMV-derived T cell epitopes. (A) Detection of several LCMVderived $\mathrm{T}$ cell epitopes by peptide-specific CTL lines. BMC cells were infected with LCMV-WE and treated with $40 \mu \mathrm{M}$ AAF-CMK (top panels, triangles) or $100 \mu \mathrm{M}$ butabindide (lower panels, triangles) overnight or left untreated (squares). Uninfected cells are marked with diamonds. Activation of CTL lines by LCMV-infected cells was analyzed by staining for CD8 and intracellular IFN $-\gamma$. Shown are the percentages of IFN- $\gamma$ positive cells of $\mathrm{CD}^{+}$cells as determined by flow cytometry. The percentage of intracellular IFN- $\gamma$ (y axis) produced by CTL lines is plotted versus the E:S ratio. The peptide specificity of the CTL lines is indicated on the top of the panels. One experiment out of four is shown. (B) TPPII activity is markedly reduced in AAFCMK- or butabindide-treated cells. BMC cells (the same cells as analyzed in Fig. 2A) were treated with $40 \mu \mathrm{M} \mathrm{AAF-CMK}$ (named $\mathrm{AAF}$ ) or $100 \mu \mathrm{M}$ butabindide (named but.) overnight. Crude lysates of these cells were assayed for hydrolysis of the TPPIIspecific fluorogenic substrate AAF-AMC. The fluorescence of the AMC leaving groups was determined after 60 min of incubation. Data represent the percentage of TPPII activity compared to untreated cells $(\varnothing)$. Values are the means \pm SD of triplicate determinations. butabindide, we analyzed cell extracts with the help of the TPPII-specific substrate Ala-Ala-Phe-7-amido4-methylcoumarin (AAF-AMC). Recently, Wherry et al. reported that purified TPPII is inhibited by AAF-CMK to $100 \%$, whereas crude lysates from inhibitor-treated cells decreased the TPPII-like activity to approximately $70 \%$ [16]. The authors proposed that the efficient inhibition of purified TPPII by AAF-CMK suggests that the residual ( $\sim 30 \%)$ AAF-AMC hydrolysis observed in cell lysates may be due to other protease activities. When we performed this assay, a similar degree of inhibition was observed in crude lysates from AAF-CMK- $(\sim 80 \%)$ and butabindide-treated ( $\sim 85 \%$ ) cells (Fig. $2 \mathrm{~B})$. Therefore, TPPII is indeed inhibited in AAF-CMK- and butabindidetreated cells but has no influence on the presentation of LCMV-derived T cell epitopes.

\section{Presentation of GP276-286 and NP396-404 is markedly reduced upon proteasome inhibition}

In order to demonstrate that our system is able to distinguish between small differences in antigen presentation, we treated LCMV-infected BMC cells with the proteasome-specific inhibitor MG132 and detected GP276-286 and NP396-404 presented on MHC class I with peptide-specific CTL. GP276-286 and NP396-404 presentation were markedly reduced after $3 \mathrm{~h}$ of proteasome inhibition (Fig. 3A, B). Therefore, we conclude that our approach to detect MHC class I presentation by ICS for IFN- $\gamma$ with peptide-specific CTL is capable to discriminate between differences in antigen presentation. It has been reported that besides GP276-286 and NP396-404, NP118-126 [17] and GP33-41 [18] are proteasome dependent, while none of these epitopes was produced by TPPII (Fig. 1, 2). Hence, the proteasome is the main protease in generating these epitopes and probably is responsible for most MHC class I ligands.

To address whether a potential excess of antigen might prevent the detection of minor differences in antigen processing, decreasing amounts of a plasmid encoding the LCMV-NP $(1.5,0.75,0.3,0.15 \mu \mathrm{g})$ were transfected into HEK-D ${ }^{\mathrm{b}}$ cells. Cells were treated with $100 \mu \mathrm{M}$ butabindide, and NP396-404 presentation was detected with peptide-specific CTL (Fig. 4). No difference could be detected between butabindide-treated and untreated cells. A fivefold reduction (from 1.5 to $0.3 \mu \mathrm{g}$ ) of NP-plasmids led to a reduction in CTL activation from $10-12 \%$ to roughly background levels. Hence, assuming that TPPII plays indeed a decisive role in the processing of LCMV-derived T cell epitopes, a reduction in peptide presentation should have been easily detected in our system (Fig. 1, 2). 
TPPII overexpression does not increase the presentation of LCMV-derived MHC class I ligands

To further clarify the impact of TPPII on the presentation of LCMV-derived epitopes, a genetic approach was chosen. It has been reported that down-regulation of TPPII activity by siRNA leads to a reduction of 50\% [16] to $60 \%$ [8]. This rather meager down-regulation by siRNA prompted us to investigate LCMV epitope
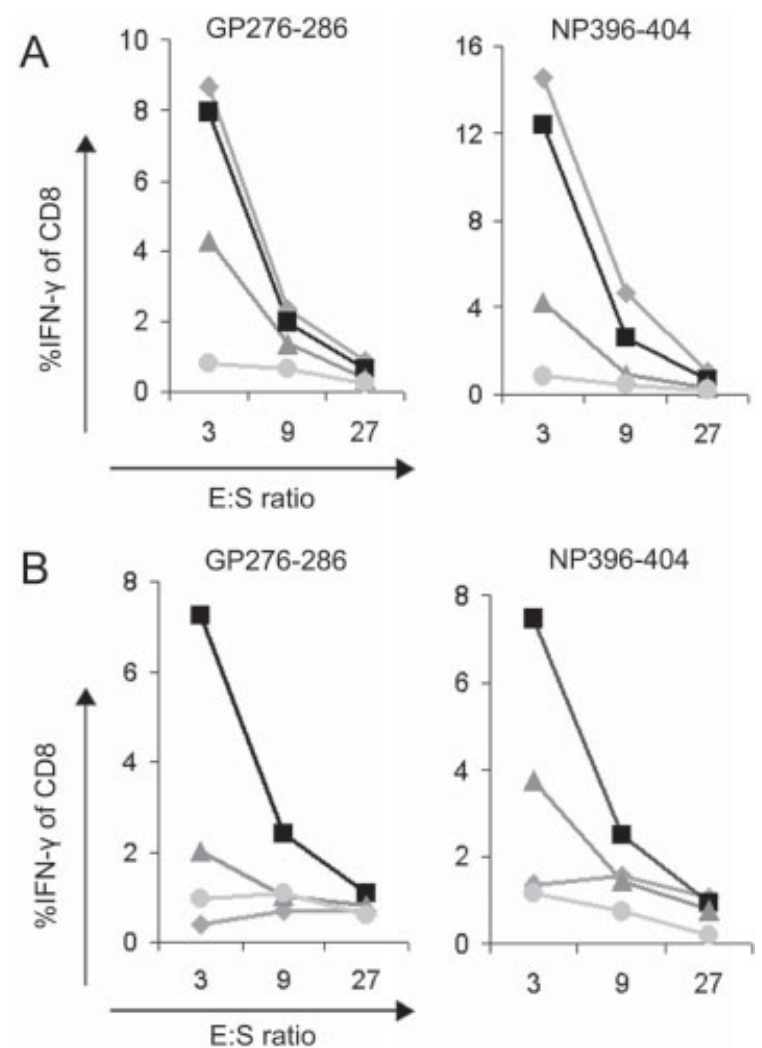

Figure 3. Proteasome inhibition significantly reduces presentation of the LCMV-derived epitopes GP276-286 and NP396-404. (A) Recovery of GP276-286 and NP396-404 after acid wash treatment during proteasome inhibition. After the acid wash procedure, LCMV-infected BMC cells were incubated for $3 \mathrm{~h}$ with $5 \mu \mathrm{M}$ MG132 (triangles) or left untreated (squares). GP276-286 or NP396-404 presentation was analyzed by activation of peptide-specific CTL lines by staining for CD8 and intracellular IFN- $\gamma$. Infected cells without acid wash treatment are indicated with diamonds and uninfected cells are marked with circles. The peptide specificity of the CTL lines used for ICS is indicated on the top of the panels. One experiment out of two is shown. (B) Recovery of GP276-286 and NP396-404 after BFA treatment during proteasome inhibition. BMC cells were infected with LCMV-WE overnight and simultaneously treated with $10 \mu \mathrm{g} / \mathrm{mL}$ BFA. BFA was removed and cells were incubated for $3 \mathrm{~h}$ with $5 \mu \mathrm{M}$ MG132 (triangles) or left untreated (squares). GP276-286 or NP396-404 presentation was analyzed by activation of peptide-specific CTL lines by staining for CD8 and intracellular IFN- $\gamma$. Infected cells in the presence of BFA are indicated with diamonds and uninfected cells are marked with circles. One experiment out of two is shown. presentation in cells transiently overexpressing TPPII. Earlier experiments have shown that overexpression is a suitable approach to investigate antigen processing when the analyzed protease plays a key role in epitope generation $[5,10,19]$. Due to low endogenous TPPII expression and high transfection efficiencies, the human embryonic kidney cell line (HEK293) was chosen. Transient rather than stable overexpression of TPPII was performed because TPPII has been reported to disturb mitotic fidelity [20]. Because of restriction element incompatibility, the HEK293 cells were stably transfected with an expression construct encoding the $\mathrm{H}-2 \mathrm{D}^{\mathrm{b}}$ restriction element (named HEK- ${ }^{\mathrm{b}}$ ) (Fig. 5A). To obtain a high transfection rate, HEK- $\mathrm{D}^{\mathrm{b}}$ cells were either transfected with plasmids encoding CD4 and TPPII or, as a negative control, with CD4 and CD14. TPPII and CD14 constructs were transfected in fourfold excess compared to $\mathrm{CD} 4$ plasmids to ascertain that every cell containing CD4 also overexpresses TPPII or CD14. CD4 ${ }^{+}$cells were magnetically enriched and CD14 expression was controlled by flow cytometry (Fig. 5B) and TPPII overexpression was analyzed by Western blot (Fig. 5C). The transfected cells were infected with LCMV-WE and the amount of antigen presented on MHC class I of cells overexpressing TPPII was analyzed with the help of GP33-, GP276-, GP92-, and NP396-specific T cell lines. To exclude that TPPII expression affects LCMV replication, the infection was controlled by surface staining of the LCMV glycoprotein (Fig. 5D). No difference of GP33, NP396 and GP92 presentation in cells overexpressing TPPII compared to control cells could be observed (Fig. 5E). In contrast, GP276 presentation was slightly

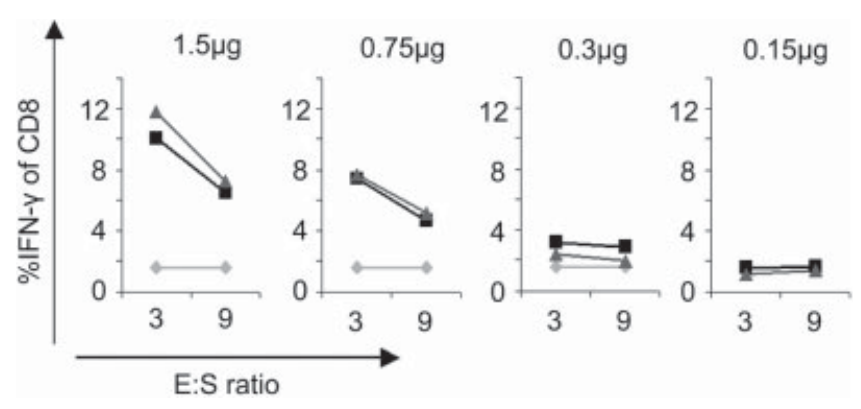

Figure 4. No influence of TPPII in NP396-404 presentation under limited peptide supply. HEK- $\mathrm{D}^{\mathrm{b}}$ cells were transiently transfected with various amounts $(1.5,0.75,0.3$ or $0.15 \mu \mathrm{g}$; indicated on the top of the panels) of a plasmid encoding the LCMV-NP and simultaneously treated with $100 \mu \mathrm{M}$ butabindide (squares) in the absence of FCS or left untreated (triangles). Non-transfected cells are marked with diamonds. Activation of NP396-specific CTL lines by NP-transfected cells was analyzed by staining for CD8 and intracellular IFN- $\gamma$. Shown are the percentages of IFN- $\gamma$-positive cells of $\mathrm{CD} 8^{+}$cells as determined by flow cytometry. The percentage of intracellular IFN- $\gamma$ (y axis) produced by CTL lines is plotted versus the E:S ratio. One experiment out of three is shown. 
decreased in TPPII-transfected cells, which is in agreement with data from AAF-CMK-treated cells (Fig. 2) which displayed an elevated GP276 presentation. A similar finding has been published by Altrich-VanLith et al., who reported on an epitope derived from tyrosinase [Tyr $\left.{ }_{369-377}(\mathrm{D})\right]$, which is enhanced upon TPPII inhibition [21]. Hence, it appears that TPPII has a destructive role for GP276 but is generally not crucial for the presentation of LCMV-derived T cell epitopes.
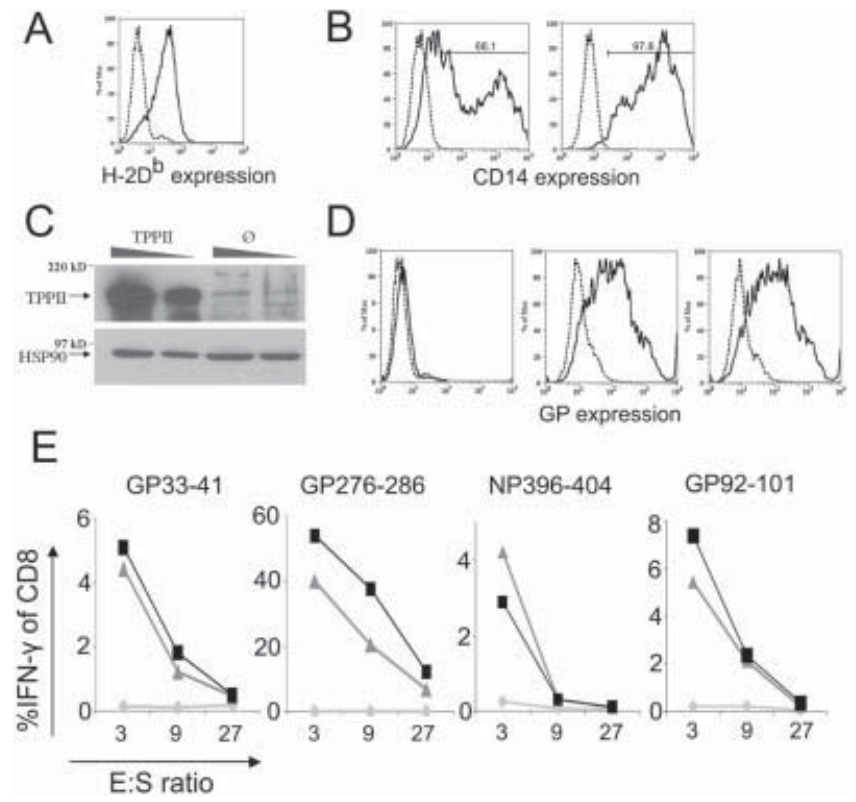

Figure 5. Marginal effect of TPPII overexpression on the presentation of LCMV-derived $\mathrm{T}$ cell epitopes. (A) Flow-cytometric analysis of $\mathrm{H}-2 \mathrm{D}^{\mathrm{b}}$ surface expression (solid line) of stably transfected HEK-D $\mathrm{D}^{\mathrm{b}}$ cells. (B) Analysis of magnetically sorted cells by flow cytometry. Magnetically sorted cells were analyzed for CD4 and the CD14 surface expression (solid lines) by flow cytometry. As a negative control, cells were not stained (dashed lines). The left panel represents transfected cells before sorting and the right panel displays MACS-purified cells. (C) Western blot analysis of TPPII overexpression. Lysates of cells overexpressing TPPII (marked with TPPII) or CD14 (indicated with $\varnothing$ ) were analyzed with a polyclonal antibody specific for TPPII. Undiluted and threefold diluted lysates were loaded. As a loading control, the same amounts of lysates were probed with an anti-HSP90 antibody. (D) Analysis of LCMV-GP surface expression (solid line) by flow cytometry. The left panel represents uninfected, the middle LCMV-infected cells overexpressing CD14, and the right panel infected cells overexpressing TPPII. (E) Detection of different LCMV-derived T cell epitopes by peptide-specific CTL lines in cells overexpressing TPPII. HEK-D ${ }^{\mathrm{b}}$ cells were transfected with CD4/TPPII (triangles) or CD4/CD14 (squares), infected with LCMV-WE, and after 2 days $\mathrm{CD}^{+}$cells were magnetically isolated and used as presenters in ICS analysis. Uninfected HEK- $\mathrm{D}^{\mathrm{b}}$ cells are displayed in diamonds. The experiments have been repeated three times, yielding similar results.

\section{Presentation of the SIINFEKL epitope of ovalbumin and the pp89/168-176 epitope of MCMV are not affected by TPPII inhibition}

In order to address whether other non-LCMV-derived MHC class I T cell epitopes were affected by TPPII, two additional peptides derived from OVA (Fig. 6) and from the murine cytomegalovirus (MCMV) (Fig. 7) were investigated. BMC cells treated with $30 \mu \mathrm{M}$ AAF-CMK or $100 \mu \mathrm{M}$ butabindide were infected with a recombinant vaccinia virus (rVV) expressing OVA. The amount of OVA-derived SIINFEKL was detected with peptidespecific CTL lines by ICS (Fig. 6A, B). No difference could be observed between TPPII-inhibited and untreated cells, although TPPII activity was reduced to $20-30 \%$ of normal activity (Fig. 6C). Similar results were obtained by York et al., showing little or no reduction in recovery of $\mathrm{H}-2 \mathrm{~K}^{\mathrm{b}}$-SIINFEKL at the cell surface after acid treatment in TPPII-inhibited cells expressing the full-length OVA. In contrast, proteasome inhibition (MG132 treatment) abolished the recovery of $\mathrm{H}-2 \mathrm{~K}^{\mathrm{b}}$-SIINFEKL at the surface of cells expressing OVA [22]. In addition to OVA-derived SIINFEKL, we tested the MCMV-derived epitope pp89/168-176. L- $\mathrm{L}^{\mathrm{d}}$ cells infected with an rVV expressing the pp89 protein were
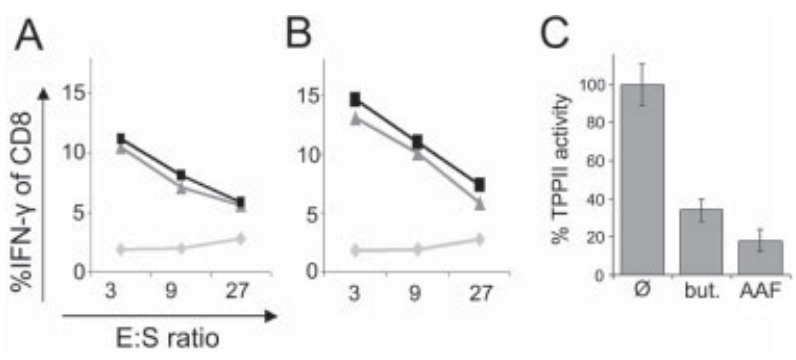

Figure 6. Marginal effect of TPPII inhibition on the presentation of OVA-derived SIINFEKL. (A, B) Detection of SIINFEKL in TPPIIinhibited cells by peptide-specific CTL lines. BMC cells were infected with VV-OVA and treated with $100 \mu \mathrm{M}$ butabindide (A) or $30 \mu \mathrm{M}$ AAF (B). Inhibitor-treated cells are marked with triangles, untreated cells with squares, and uninfected cells with diamonds. Activation of CTL lines by LCMV-infected cells was analyzed by staining for CD8 and intracellular IFN- $\gamma$. Shown are the percentages of IFN- $\gamma$-positive cells of $\mathrm{CD} 8^{+}$cells as determined by flow cytometry. The percentage of intracellular IFN- $\gamma$ (y axis) produced by CTL lines is plotted versus the $\mathrm{E}: \mathrm{S}$ ratio. The peptide specificity of the CTL lines is indicated on the top of the panels. One experiment out of three is shown. (C) TPPII activity is markedly reduced in AAF-CMK- or butabindide-treated cells. BMC cells (the same cells as analyzed in Fig. 6A, B) were treated with $100 \mu \mathrm{M}$ butabindide (named but.) or $30 \mu \mathrm{M}$ AAF-CMK (named AAF) for $4 \mathrm{~h}$. Crude lysates of these cells were assayed for hydrolysis of the TPPIIspecific fluorogenic substrate AAF-AMC. The fluorescence of the AMC leaving groups was determined after $60 \mathrm{~min}$ of incubation. Data represent the percentage of TPPII activity compared to untreated cells $(\varnothing)$. Values are the means \pm SD of triplicate determinations. 

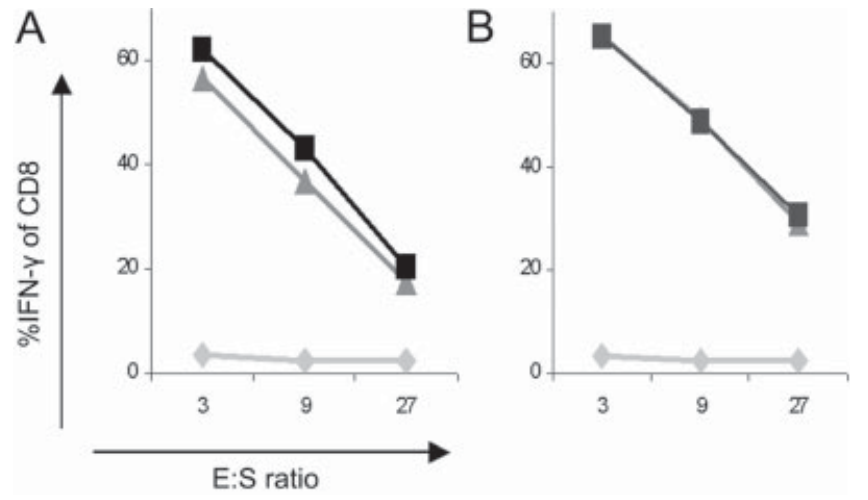

Figure 7. Presentation of MCMV pp89/168-176 is not affected by TPPII. (A, B) Detection of pp89/168-176 in TPPII-inhibited cells by peptide-specific CTL lines. L-L ${ }^{\mathrm{d}}$ cells were infected with VVpp89 and treated with $100 \mu \mathrm{M}$ butabindide (left side) or $30 \mu \mathrm{M}$ AAF (right side). Inhibitor-treated cells are marked with triangles, untreated cells with squares and uninfected cells with diamonds. Activation of CTL lines by LCMV-infected cells was analyzed by staining for $\mathrm{CD} 8$ and intracellular IFN- $\gamma$. Shown are the percentages of IFN- $\gamma$-positive cells of $\mathrm{CD}^{+}$cells as determined by flow cytometry. The percentage of intracellular IFN- $\gamma$ (y axis) produced by CTL lines is plotted versus the $E: S$ ratio. The peptide specificity of the CTL lines is indicated on the top of the panels. One experiment out of three is shown.

treated with $30 \mu \mathrm{M}$ AAF-CMK or $100 \mu \mathrm{M}$ butabindide. The amount of peptides presented on MHC class I was detected with peptide-specific CTL lines against pp89/ 168-176 (Fig. 7). No difference could be observed in butabindide- and AAF-CMK-treated cells compared to untreated cells. These results demonstrate that TPPII plays no essential role in generating these ligands for MHC class I molecules.

\section{Discussion}

Several cytosolic proteases have lately been discovered to influence the generation of cytotoxic $\mathrm{T}$ cell epitopes. However, the portion of epitopes positively affected by these peptidases seems to be rather low. There is little doubt that the proteasome is the main protease in processing ligands for MHC class I molecules. The proteasome can directly produce peptides in their final form or as N-terminally extended precursors. Cells treated with proteasome inhibitor for extended time periods markedly up-regulated TPPII, which prevented the accumulation of polyubiquitylated proteins [23, 24]. Hence, TPPII can compensate for proteasome function, suggesting a role for TPPII in the production of MHC class I ligands. Recently, Wherry et al. demonstrated that the generation of the influenza epitope $\mathrm{NP}_{147-155}$ is independent of TPPII in proteasome inhibitor-adapted cells [16]. TPPII possesses a low endoproteolytic activity and should therefore be capable to directly produce ligands for MHC class I molecules with the correct C terminus [6]. Using internally quenched fluorescent peptides, Reits et al. confirmed the endoproteolytic activity of TPPII [8]. In the same study, the authors proposed that a major proportion of proteasomal products is larger than 15 aa and requires further degradation by TPPII before qualifying as MHC class I ligands. This study was essentially based on the degradation of an N- or C-terminally extended model peptide after microinjection into intact cells, but the data may not be representative for peptides with different primary structures. Nevertheless, they showed that inhibition of TPPII by butabindide in Mel JuSo cells and peripheral blood lymphocytes reduced the recovery of MHC class I surface expression after acid wash treatment to the same extent as the proteasome inhibitor lactacystin. Therefore, one can assume that the bulk of CTL epitopes should be affected by TPPII. Our work could not support this intriguing concept. We analyzed several LCMV-specific Tcell epitopes (Fig. 1, 2, 5) as well as two epitopes derived from MCMV (pp89/168-176) (Fig. 7) and OVA (SIINFEKL) (Fig. 6) and found only minor effects of TPPII on the processing of ligands for MHC class I molecules. A recent study investigating the role of TPPII in trimming N-terminally extended precursors of the OVA epitope SIINFEKL came to similar conclusions [22]. Eliminating TPPII from human cells did not decrease the overall supply of peptides to MHC class I molecules and only modestly reduced the presentation of OVA-derived SIINFEKL, while treatment with proteasome inhibitors reduced these processes dramatically. Interestingly, the same authors reported that TPPII appears to be the major peptidase capable of processing proteasome products longer than 14 aa, but nevertheless, in vivo relatively few such peptides are released by the proteasome. It appears that TPPII acts only on a very limited number of CTL epitopes. In vitro proteasome digests revealed that approximately one fifth of all proteasome products are too large for direct presentation [12]. If TPPII is indeed responsible for further processing all these products for MHC class I presentation, some of our investigated LCMV-specific $\mathrm{T}$ cell epitopes or other epitopes should be affected. So far, only one epitope has been described that can directly be processed by TPPII in a proteasome-independent manner [7]. We propose that the main function of TPPII is not the processing of CTL epitopes, but rather the destruction of longer peptides to smaller fragments, which can be further digested to amino acids by other amino peptidases. In fact, York et al. reported a small but consistent increase in peptide supply to MHC class I after TPPII knockdown, presumably because TPPII destroys some peptides suitable for MHC class I presentation [22]. While processing longer peptides, in rare cases, TPPII can generate fragments convenient 
for antigen presentation, like Levy et al. [14] have shown in vitro. Our results show that TPPII has no major impact on generating six LCMV epitopes and two other peptides derived from OVA and MCMV, and therefore indicate that the effect of TPPII on antigen presentation has recently been overestimated $[8,25]$.

\section{Material and methods}

\section{Mice, viruses, cells, and media}

C57BL/6 mice $\left(\mathrm{H}-2^{\mathrm{b}}\right)$ and $\mathrm{BALB} / \mathrm{c}$ mice were originally obtained from Charles River, Germany, and were kept in a specific pathogen-free facility and used at $6-10 \mathrm{wk}$ of age. Animal experiments were approved by the review board of the Regierungspräsidium Freiburg. rVV encoding the LCMV-NP (rVVYN4) (D. Bishop, Institute of Virology, Oxford, UK), the pp89 protein [26], or the OVA protein (J. Yewdell, Bethesda, MD) were propagated on BSC40 cells. LCMV-WE was originally obtained from F. Lehmann-Grube (Hamburg, Germany) and propagated on the fibroblast line L929. Mice were infected with 200 PFU LCMV-WE i.v., and CTL were used as effector cells at day 8 post infection. HEK293 is a human embryonic kidney cell line. BMC-2 (H-2 $\left.{ }^{\mathrm{b}}\right)$ and $\mathrm{J} 774\left(\mathrm{H}-2^{\mathrm{d}}\right)$ are macrophage and DC2.4 ( $\left.\mathrm{H}-2^{\mathrm{b}}\right)$ dendritic cell lines, respectively (a kind gift from Dr. K. Rock, University of Massachusetts Medical School, Worcester, MA). D2 are methylcholanthreneinduced fibrosarcomas of B10.D2 origin. T2- $\mathrm{L}^{\mathrm{d}}$ is the TAPdeficient human lymphoblastoid T2 cell line transfected with $\mathrm{H}-2 \mathrm{~L}^{\mathrm{d}}$.

All media were purchased from Invitrogen-Life Technologies (Karlsruhe, Germany) and contained GlutaMAX, 10\% FCS, and $100 \mathrm{U} / \mathrm{mL}$ penicillin/streptomycin.

\section{Synthetic peptides}

The synthetic peptides GP33-41 (KAVYNFATC), GP92-101 (CSANNSHHYI), GP276-286 (SGVENPGGYCL), NP205-212 (YTVKYPNL), NP396-404 (FQPQNGQFI), NP118-126 (RPQASGVYM), pp89/168-176 (YPHFMPTNL) and OVASIINFEKL were obtained from Eurogentec (Cologne, Germany).

\section{Transfection}

HEK293 cells were transfected with an expression plasmid encoding $\mathrm{H}-2 \mathrm{D}^{\mathrm{b}}$ (a kind gift from F. Momburg, Heidelberg, Germany). Cells were plated to $80 \%$ confluence and were stably transfected with $\mathrm{H}-2 \mathrm{D}^{\mathrm{b}}$ and puromycin resistance plasmids, according to the manufacturer's protocol (FuGENE 6; Roche, Basel, Switzerland). Clonal and selection drug-resistant cells were tested for $\mathrm{H}-2 \mathrm{D}^{\mathrm{b}}$ expression by flow cytometry.

HEK- $\mathrm{D}^{\mathrm{b}}$ cells were extensively washed and transiently transfected (FCS-free conditions) with an expression plasmid encoding the LCMV-NP [27] using FuGENE 6 (3 $\mu \mathrm{L}$ FuGene; $1.5,0.75,0.3$ or $0.15 \mu \mathrm{g}$ plasmid DNA) according to the manufacturer's instructions. At $1 \mathrm{~h}$ post infection, cells were incubated with $100 \mu \mathrm{M}$ butabindide in the absence of FCS. Cells were analyzed for peptide presentation $20 \mathrm{~h}$ post transfection.

\section{Western blotting}

TPPII-transfected and untransfected HEK- $\mathrm{D}^{\mathrm{b}}$ cells were lysed in $150 \mu \mathrm{L}$ lysis buffer (50 mM Tris pH 8, 2 mM EDTA, 1\% Triton X-100, $5 \mu \mathrm{g} / \mathrm{mL}$ aprotinin, $1 \mathrm{mM}$ phenylmethylsulfonyl fluoride, $4 \mu \mathrm{M}$ pepstatin, and $5 \mu \mathrm{g} / \mathrm{mL}$ leupeptin) on ice for $15 \mathrm{~min}$. Proteins were separated by SDS-PAGE ( $8 \%$ gel), blotted onto nitrocellulose (Schleicher \& Schuell BioSciences, Dassel, Germany), blocked (PBS/5\% w/v low-fat dry milk/ $0.2 \%$ Tween-20) for $1 \mathrm{~h}$ and agitated overnight at $4{ }^{\circ} \mathrm{C}$ with a chicken anti-TPPII antibody (1 : 2000; Immunsystem, Uppsala, Sweden). The blots were washed three times and incubated for $2 \mathrm{~h}$ with peroxidase-conjugated rabbit anti-chicken antibody (1 : 3000; Sigma, Germany). After extensive washing with PBS/0.2\% Tween-20, proteins were visualized on X-ray films by enhanced chemiluminescence.

\section{Antibodies and inhibitors}

KL25 is a mouse mAb reactive with the LCMV glycoprotein [28]. The mAb KH95 (BD Pharmingen, San Diego, CA) reacts with the $\mathrm{H}-2 \mathrm{D}^{\mathrm{b}}$ MHC class I molecule. Chicken anti-human TPPII (Immunsystem) recognizes mouse and human TPPII. CD14-FITC and CD4-FITC were acquired from Miltenyi Biotec.

Butabindide (Tocris, Avonmouth, UK) was applied on intensively washed cells in the absence of FCS and used at a concentration of $100 \mu \mathrm{M}$. AAF-CMK (Bachem, Germany) was used at a concentration of 20,30 , or $40 \mu \mathrm{M}$, and MG132 (Sigma, Germany) at a concentration of $5 \mu \mathrm{M}$.

\section{Enzyme assay}

Determination of TPPII activity in cell lysates with the help of the fluorogenic substrate AAF-AMC (Sigma, Germany) was conducted as previously detailed [16].

\section{Flow cytometry}

Flow cytometry was performed exactly as previously described [29].

\section{ICS for IFN- $\gamma$}

Stimulator cells $\left(1.5 \times 10^{5}\right.$ to $\left.4 \times 10^{5}\right)$ were incubated in round-bottom 96-well plates with $4 \times 10^{5}$ to $4.5 \times 10^{5}$ CTL in $200 \mu \mathrm{L}$ IMDM $10 \%$ + brefeldin A (BFA) $(10 \mu \mathrm{g} / \mathrm{mL})$ for $4 \mathrm{~h}$ at $37^{\circ} \mathrm{C}$. The staining, fixation and permeabilization of the cells were performed exactly as detailed previously [29].

\section{Peptide-specific CTL lines}

LCMV, pp89/168-176, and OVA-SIINFEKL peptide-specific CTL-lines were generated exactly as previously described [30]. LCMV- and OVA-SIINFEKL peptide-specific CTL lines were restimulated with peptide-loaded DC2.4 cells; and pp89/ 168-176-specific CTL with peptide-loaded D2 cells. CTL were 
used in ICS at an effector-to-stimulator (E:S) ratio of 1-3 in the first dilution, and serial threefold dilutions of stimulators were performed.

\section{TPPII overexpression}

HEK-D ${ }^{\mathrm{b}}$ cells were transfected with either plasmids encoding TPPII and CD4 (4:1) or CD14 and CD4 (4:1) according to the manufacturer's protocol (FuGENE 6; Roche). At $6 \mathrm{~h}$ post transfection, cells were infected with LCMV-WE (MOI of 0.1) and cultured overnight. CD4 ${ }^{+}$cells were positively selected using anti-CD4 antibody-conjugated magnetic microbeads (Miltenyi Biotec). Transfection and sorting efficiency was assessed by flow cytometry and achieved $\sim 95 \%$ CD $14^{+}$cells. Cells were cultured overnight in the presence of IFN- $\gamma$ (100 U/ $\mathrm{mL})$ and TNF- $\alpha(200 \mathrm{U} / \mathrm{mL})$ to increase MHC expression, and antigen presentation was analyzed with the help of peptidespecific CTL lines. Infection efficiency was controlled by flow cytometry (GP surface staining) and TPPII overexpression was analyzed by Western blot.

Acknowledgements: Dr. Birgitta Tomkinson is acknowledged for supplying the TPPII expression plasmid. This work was funded by the German National Science Foundation (DFG) Grant No. GR 1517/4-2. The authors have no conflicting financial interests.

\section{References}

1 Kisselev, A. F., Akopian, T. N. and Goldberg, A. L., Range of sizes of peptide products generated during degradation of different proteins by archaeal proteasomes. J. Biol. Chem. 1998. 273: 1982-1989.

2 Beninga, J., Rock, K. L. and Goldberg, A. L., Interferon-gamma can stimulate post-proteasomal trimming of the $\mathrm{N}$ terminus of an antigenic peptide by inducing leucine aminopeptidase. J. Biol. Chem. 1998. 273: 18734-18742.

3 Stoltze, L., Schirle, M., Schwarz, G., Schroter, C., Thompson, M. W., Hersh, L. B., Kalbacher, H. et al., Two new proteases in the MHC class processing pathway. Nat. Immunol. 2000. 1: 413-418.

4 Saric, T., Beninga, J., Graef, C. I., Akopian, T. N., Rock, K. L. and Goldberg, A. L., Major histocompatibility complex class I-presented antigenic peptides are degraded in cytosolic extracts primarily by thimet oligopeptidase. J. Biol. Chem. 2001. 276: 36474-36481.

5 York, I. A., Mo, A. X. Y., Lemerise, K., Zeng, W. Y., Shen, Y. L., Abraham, C. R., Saric, T. et al., The cytosolic endopeptidase, thimet oligopeptidase, destroys antigenic peptides and limits the extent of MHC class I antigen presentation. Immunity 2003. 18: 429-440.

6 Geier, E., Pfeifer, G., Wilm, M., Lucchiari-Hartz, M., Baumeister, W., Eichmann, K. and Niedermann, G., A giant protease with potential to substitute for some functions of the proteasome. Science 1999. 283: 978-981.

7 Seifert, U., Maranon, C., Shmueli, A., Desoutter, J. F., Wesoloski, L., Janek, K., Henklein, P. et al., An essential role for tripeptidyl peptidase in the generation of an MHC class I epitope. Nat. Immunol. 2003. 4: 375-379.

8 Reits, E., Neijssen, J., Herberts, C., Benckhuijsen, W., Janssen, L., Drijfhout, J. W. and Neefjes, J., A major role for TPPII in trimming proteasomal degradation products for MHC class I antigen presentation. Immunity 2004. 20: 495-506.

9 York, I. A., Chang, S. C., Saric, T., Keys, J. A., Favreau, J. M., Goldberg, A. L. and Rock, K. L., The ER aminopeptidase ERAP1 enhances or limits antigen presentation by trimming epitopes to 8-9 residues. Nat. Immunol. 2002. 3: 1177-1184.
10 Saric, T., Chang, S. C., Hattori, A., York, I. A., Markant, S., Rock, K. L., Tsujimoto, M. et al., An IFN-gamma-induced aminopeptidase in the ER, ERAP1, trims precursors to MHC class I-presented peptides. Nat. Immunol. 2002. 3: 1169-1176.

11 Saveanu, L., Carroll, O., Lindo, V., Del Val, M., Lopez, D., Lepelletier, Y., Greer, F. et al., Concerted peptide trimming by human ERAP1 and ERAP2 aminopeptidase complexes in the endoplasmic reticulum. Nat. Immunol. 2005. 6: 689-697.

12 Kisselev, A. F., Akopian, T. N., Woo, K. M. and Goldberg, A. L., The sizes of peptides generated from protein by mammalian 26 and $20 \mathrm{~S}$ proteasomes Implications for understanding the degradative mechanism and antigen presentation. J. Biol. Chem. 1999. 274: 3363-3371.

13 Rockel, B., Peters, J., Muller, S. A., Seyit, G., Ringler, P., Hegerl, R., Glaeser, R. M. et al., Molecular architecture and assembly mechanism of Drosophila tripeptidyl peptidase II. Proc. Natl. Acad. Sci. USA 2005. 102: 10135-10140.

14 Levy, F., Burri, L., Morel, S., Peitrequin, A. L., Levy, N., Bachi, A., Hellman, U. et al., The final N-terminal trimming of a subaminoterminal proline-containing HLA class I-restricted antigenic peptide in the cytosol is mediated by two peptidases. J. Immunol. 2002. 169: 4161-4171.

15 Khan, S., de Giuli, R., Schmidtke, G., Bruns, M., Buchmeier, M., van den Broek, M. and Groettrup, M., Cutting Edge: Neosynthesis is required for the presentation of a T cell epitope from a long lived viral protein. $J$. Immunol. 2001. 167: 4801-4804.

16 Wherry, E. J., Golovina, T. N., Morrison, S. E., Sinnathamby, G., McElhaugh, M. J., Shockey, D. C. and Eisenlohr, L. C., Re-evaluating the generation of a "proteasome-independent" MHC class I-restricted CD8 T cell epitope. J. Immunol. 2006. 176: 2249-2261.

17 Schwarz, K., de Giuli, R., Schmidtke, G., Kostka, S., van den Broek, M., Kim, K., Crews, C. M. et al., The selective proteasome inhibitors lactacystin and expoxomicin can be used to either up- or downregulate antigen presentation at non-toxic doses. J. Immunol. 2000. 164: 6147-6157.

18 Gallimore, A., Schwarz, K., van den Broek, M., Hengartner, H. and Groettrup, M., The proteasome inhibitor lactacystin prevents the generation of an endoplasmic reticulum leader-derived T cell epitope. Mol. Immunol. 1998. 35: 581-591.

19 Groettrup, M., Soza, A., Eggers, M., Kuehn, L., Dick, T. P., Schild, H., Rammensee, H.-G. et al., A role for the proteasome regulator PA28 $\alpha$ in antigen presentation. Nature 1996. 381: 166-168.

20 Stavropoulou, V., Xie, J., Henriksson, M., Tomkinson, B., Imreh, S. and Masucci, M. G., Mitotic infidelity and centrosome duplication errors in cells overexpressing tripeptidyl-peptidase II. Cancer Res. 2005. 65: 1361-1368.

21 Altrich-VanLith, M. L., Ostankovitch, M., Polefrone, J. M., Mosse, C. A., Shabanowitz, J., Hunt, D. F. and Engelhard, V. H., Processing of a class Irestricted epitope from tyrosinase requires peptide $\mathrm{N}$-glycanase and the cooperative action of endoplasmic reticulum aminopeptidase 1 and cytosolic proteases. J. Immunol. 2006. 177: 5440-5450.

22 York, I. A., Bhutani, N., Zendzian, S., Goldberg, A. L. and Rock, K. L., Tripeptidyl peptidase II is the major peptidase needed to trim long antigenic precursors, but is not required for most MHC class I antigen presentation. $J$. Immunol. 2006. 177: 1434-1443.

23 Glas, R., Bogyo, M., McMaster, J. S., Gaczynska, M. and Ploegh, H. L., A proteolytic system that compensates for loss of proteasome function. Nature 1998. 392: 618-622.

24 Wang, E. W., Kessler, B. M., Borodovsky, A., Cravatt, B. F., Bogyo, M., Ploegh, H. L. and Glas, R., Integration of the ubiquitin-proteasome pathway with a cytosolic oligopeptidase activity. Proc. Nat. Acad. Sci. USA 2000. 97: 9990-9995.

25 Kloetzel, P. M., Generation of major histocompatibility complex class I antigens: Functional interplay between proteasomes and TPPII. Nat. Immunol. 2004. 5: 661-669.

26 Volkmer, H., Bertholet, C., Jonjic, S., Witter, R. and Koszinowski, U., Cytolytic T lymphocyte recognition of the murine cytomegalovirus nonstructural immediate-early protein pp89 expressed by recombinant vaccinia virus. J. Exp. Med. 1987. 166: 668-677.

27 Rodriguez, F., Zhang, J. and Whitton, J. L., DNA immunization: Ubiquitination of a viral protein enhances cytotoxic T-lymphocyte induction and antiviral protection but abrogates antibody induction. J. Virol. 1997. 71: 8497-8503. 
28 Bruns, M., Cihak, J., Müller, G. and Lehmann-Grube, F., Lymphocytic choriomeningitis virus. VI. Isolation of a glycoprotein mediating neutralization. Virology 1983. 130: 247-251.

29 Basler, M., Youhnovski, N., Van Den Broek, M., Przybylski, M. and Groettrup, M., Immunoproteasomes down-regulate presentation of a subdominant T cell epitope from lymphocytic choriomeningitis virus. $J$. Immunol. 2004. 173: 3925-3934.

30 Basler, M., Moebius, J., Elenich, L., Groettrup, M. and Monaco, J. J., An altered T cell repertoire in MECL-1-deficient mice. J. Immunol. 2006. 176: 6665-6672. 